\title{
GHHECR Protocol for Energy Conserving Routing in Sensor Networks
}

\author{
Mohd. Uruj Jaleel \\ Research Scholar \\ Dept. of Computer Science \\ Singhania University, India
}

\author{
Dr. Ashish Chaturvedi \\ Assistant Professor \\ Dept. of Applied Science \& \\ Humanities, GBIT,Meerut
}

\begin{abstract}
Energy management is the prime issue in the domain of Sensor networks where the deployment process requires a continuous resource of energy. Most of the energy required during communication over the network results a need of energy conserving routing protocols in sensor networks. This paper demonstrates a new protocol GHHECR (Group Head Hierarchical Energy Conserving Routing) aiming of maximizing the network lifetime. The article forms in this way that firstly, it explores the neural network based determination of Group Head amongst the competitive sensor nodes and further it extends to our proposed protocol.
\end{abstract}

\section{Keywords}

Senor Networks, Energy Conserving Routing Protocol, Neural Networks.

\section{INTRODUCTION}

Wireless Sensor Networks (WSNs) comes under wireless ad hoc networks in which sensor nodes collect, process, and communicate data acquired from the physical environment to an external Base-Station (BS). Some of them are capable of sensing a special phenomenon in the environment and send the data back to one or several base stations. A quality of WSN that makes it unique is its flexibility in terms of the shape of the network and mobility of the sensor nodes. WSN can be deployed in areas where regular sensor networks (even wired networks) cannot operate. Also the self-shaping feature of WSN, along with the freedom of the wireless sensors movement makes it an ideal tool for the situations where the sensors are mobile. Today the application of sensor networks can be seen in different aspects of our lives; it is successfully applied in medical applications, military purposes, disaster area monitoring, etc $[1,2]$.

But these networks are facing various challenges such as, sensor nodes in WSN are normally battery-powered, and hence energy has to be carefully utilized in order to avoid early termination of sensors' lifetimes [3]. Since wireless sensors are not physically connected to any central resource of energy, they are completely dependent on their battery source to operate; also wireless sensors positions are not determined prior to the network deployment, thus sensors should be able to operate in a way that can automatically generate an optimal routing path and deliver the sensed information back to the base-station. Base-station integrates the received data and applies a process over it and sends the results to the user or for further processing.

Each wireless sensor node is physically not connected to any source of energy, and thus its own battery is the only dependable power supply for it. Sensor nodes are also constrained on bandwidth. Considering these two limitations, it is necessary to design routing and sensing algorithms that use innovative methods to preserve the energy of the sensors [4]. Since the lifetime of the network is highly dependent on the lifetime of the sensor's batteries [5]. The lifetime of the network can only increase by preserving the energy in the sensor nodes. Number of techniques has been evolved to increase the lifetime of the wireless sensor network. Since most of the energy consumption of each node is due to sensing and routing operations, many of the proposed techniques try to optimize these two tasks. Some approaches update the routing path when a sensor node in a path is low in energy [6] thus that they would exclude the node from the routing path and preserve its energy. Many techniques such as MCFA, GBR and Rumor routing use the shortest path method to reduce the communication and energy consumption. Many of WSN management techniques use an agent-based method to manage the wireless sensor network and its energy consumption [7- 11].

The characteristic configuration of a sensor node in a wireless sensor networks includes single or multiple sensing elements, a data processing element, communication elements and an energy source of limited energy capacity. The sensing elements perform measurements corresponds to the conditions existing at its surrounding environment. These measurements are transformed into corresponding electric signals and are processed by the processing unit. The sensor node communicates using communication elements by transmitting the data, over a wireless channel, to a specific sink point, known as base station. The base station collects the data transmitted to it by communication elements and further act as a supervisory control processor or an access point for a human interface or even as a gateway to other networks.

Today, sensor networks supports a variety of applications including environment and habitat monitoring, surveillance and reconnaissance, home automation, biomedical applications, object tracking, traffic control, fire detection, inventory control, 
agriculture, machine failure diagnosis and energy management [12-18]. Research on the network layer of the protocol basically focuses on the achievement of energy efficient route setup and consistent data communication from the sensor nodes to the base station aiming to extend the network lifetime [16]; that is why routing protocols in wireless sensor network's objective of attaining energy conservation.

\section{GROUP HEAD SELECTION USING NEURAL NETWORKS}

Sensor nodes are initially powered by batteries with full capacities. Each sensor collects data which are typically associated with other sensors in its neighborhood, and then the associated data is sent to the Base Station through Group Head for evaluating the tasks more efficiently. Assuming the periodic sensing of same period for all the sensors and Group Head is selected as in [19]. Inside each fixed group of nodes, a node is periodically elected to act as Group Head through which communication to/from Group nodes takes place.

The set of Group Head nodes can be selected on the basis of the routing cost metric explored by the equation

$$
R_{C M}=\frac{E_{k}}{A_{r}\left\{E^{T}\left(N_{k}^{S}, N_{m}^{D}\right)+E^{R}\left(N_{k}^{S}, N_{m}^{D}\right)\right\}}
$$

Where, $E_{k}$ be the energy associated with the delivery ratio of the packet, delivered correctly from source node $N^{S}$ to the destination node $N^{D}, E^{T}\left(N_{k}^{S}, N_{m}^{D}\right)$ is the energy transmitted from $N^{S}$ and $E^{R}\left(N_{k}^{S}, N_{m}^{D}\right)$ is the energy received at $N^{D}, A_{r}$ be the range area of the network.

The densely populated areas of the network will be overcrowded with Group Head nodes, while the barely populated areas will be left without any Group Head node. In such a situation, it is likely that the high cost sensors from poorly covered areas will have to perform expensive data transmissions to distant Group Head nodes which will further reduce their lifetime.

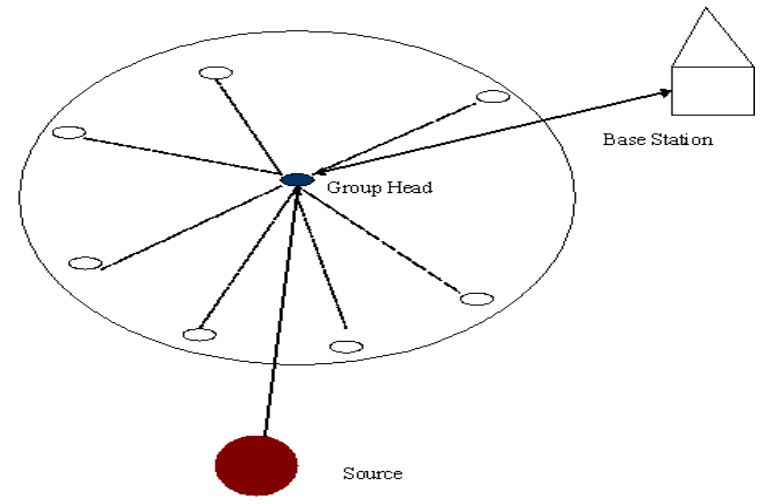

Figure 1 Layout of a Simple WSN
We are using here, five layered feed forward neural network architecture system just like in figure 2 . We have provided input patterns in form of the sensor nodes competing for Group Head.

The node with smallest value of $E_{k}$ is selected as Group Head.

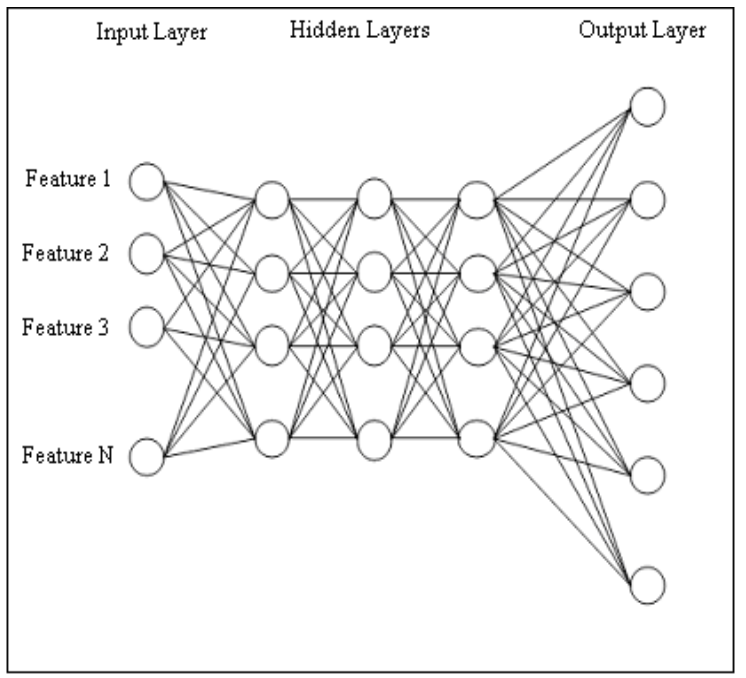

Figure 2 Architecture of FFNN

\section{GROUP HEAD HIERARCHICAL ENERGY EFFICIENT ROUTING (GHHECR) PROTOCOL}

The article proposes an energy conserving routing protocol named GHHECR, which aims the achievement of energy conservation through the use of both an energy efficient routing strategy and energy aware route selection scheme. The protocol assumed to the existence of a base station and surrounding randomly distributed sensor nodes. The base station is located at a far distant position away from the sensor field. Both the base station and the set of the sensor nodes are assumed to be stationary. It is further assumed that the base station is able to transmit with high enough power to all the network nodes, due to its unlimited power supply. However, the network nodes are supposed to be energy constrained. All the network nodes are dynamically grouped. One of the nodes within every group is elected to be the group head. Those group heads located close enough to the network base station and are capable of direct transmission to the base station with reasonable energy expenditure can be defined as Top Level group head and those group heads which are far located to the base station can supposed to be the Below Level group heads. The selection of a Group head is not random rather it is more precise. It is based on this policy that the node having the maximum residual energy will be selected as group head. The network routing architecture is shown in Figure 3. Designing of such the network routing architecture must ensure that the performance should not be affected if the size of the network increases. If we look at this architecture we can found that all the nodes even far located, can 
be able to make the proper communication with the base station through their neighboring nodes via Group heads of below level to top level.

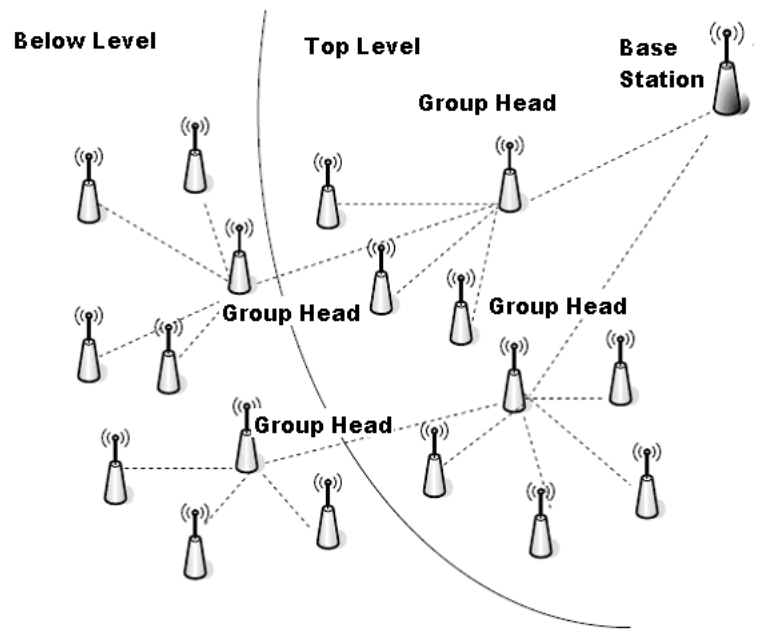

Figure 3 Network Architecture

The algorithmic steps for GHHECR protocols can be depicted as:

Step 1: First of all, the nodes must on their receivers.

Step 2: The nodes have to send their advertisement message through a TDMA (Time Division Multiple Access) schedule for providing the information about network field to the base station using the same transmission energy in a specific time allotted to them.

Step 3: Receiver nodes which receive this advertisement message estimate their comparative distance from this node, according to the power of the advertisement signal received.

Step 4: The base station inbuilt with neural network system selects some of the nodes from which it has received an advertisement message to be the Top level group heads as per the mechanism described in last section. Similarly, it also selects some of the nodes from which it has not received any advertisement message to be the below level group heads.

Step 5: Then base station broadcasts the identities (IDs) to the newly selected group heads.

Step 6: Each non group head node selects for the current round to participate for group head, whose advertisement message had been received before with the largest signal strength.

Step 7: Since lower level group head nodes cannot communicate directly with the base station, it is necessary for them to route their messages via a path consisting of a Top level group head node and/or via other below level group head nodes located closer to the top level. In this way however, there are various alternative paths that may me followed, but we will consider here only those which are having minimum residual energy after communicating the message with base station.

Step 8: The nodes of the group informs to their corresponding group using a CSMA (Carrier-Sense Multiple Access) MAC protocol. In this way, group heads receives all the messages from the nodes that want to be included in their group and creates a TDMA schedule according to the size of their group.

Step 9: Then each group head broadcasts this schedule back to the nodes in its group, in order to inform each node when it can transmit. Thus, the data transmission may start. During its allocated transmission time, each node sends to its group head quantitative data concerning the sensed events.

Step 10: As all the data have been received to the group heads by their group nodes, each group head performs signal processing functions to aggregate the data it has received along with its own data into a single composite message. This message consists of ID of the node having the highest residual energy among the group nodes, along with the id of the node that has sensed it.

Step 11: After each group head has created its aggregate message, it waits until its own time slot in order to transmit this aggregate message to the base station either directly, if this is possible, or via intermediate upper level cluster heads.

Step 12: The base station collects all the messages transmitted to it by group heads of the network and determines the new group heads by using the data of the received messages. More specifically, the node having the highest residual energy in each group is selected to be the new group head.

Step 13: Finally, the base station broadcasts the IDs of the newly selected group heads.

Consequently, each non group head node chooses the group it intends to belong to. The steady state phase is further carried on as a continuously recurrent execution of the procedures from step 6 to step 13.

\section{CONCLUSION}

In this paper we have proposed an energy conserving routing protocol named GHHECR, which aims the achievement of energy conservation through the use of both an energy efficient routing strategy and energy aware route selection scheme. Neural network system has been successfully applied to elect the group head from the competitive nodes. In this paper, we used arbitrary data for our experiment of neural network based group head selection; it is also expected to generate a real time data for the experiment in future and to carry an experiment to test our proposed algorithm. 


\section{ACKNOWLEDGEMENT}

I take this immense opportunity to express my sincere gratitude towards my supervisor Dr. Ashysh Chaturvedi for his invaluable guidance. I consider myself extremely fortunate to have a chance to work under his supervision. I like to pay my sincere thanks to him, for their kind support and cooperation.

\section{REFERENCES}

[1] Akyildiz, I.F.; Weilian Su; Sankarasubramaniam, Y. Cayirci, E., "A survey on sensor networks", Communications Magazine, IEEE, vol.40, no.8, pp. 102114, Aug 2002.

[2] Chee-Yee Chong; Kumar, S.P., "Sensor networks: evolution, opportunities, and challenges", Proceedings of the IEEE, vol.91, no.8, pp. 1247-1256, Aug. 2003.

[3] N. Al-Karaki, A.E. Kamal, "Routing techniques in wireless sensor networks: a survey", IEEE Wireless Communications, 11:6, 6-28, 2004.

[4] Al-Karaki, J.N.; Kamal, A.E., "Routing techniques in wireless sensor networks: a survey", Wireless Communications, IEEE, vol.11, no.6, pp. 6-28, Dec. 2004.

[5] Heinzelman, W.; Chandrakasan, A.; and Balakrishnan, H.; "Energy- Efficient Communication Protocol for Wireless Microsensor Networks", Proceedings of the 33rd Hawaii International Conference on System Sciences (HICSS '00), January 2000.

[6] Li Hu; Yun Li; Qinbin Chen; Jia-yi Liu; Ke-ping Long, "A New Energy-Aware Routing Protocol for Wireless Sensor Networks", International Conference on Wireless Communications, Networking and Mobile Computing (WiCom 2007), pp.2444-2447, 21-25 Sept. 2007.

[7] Hosseingholizadeh, A.; Abhari, A.; "A new Agent-Based Solution for Wireless Sensor networks Management", 12th Communications and Networking Simulation Symposium (CNS), San Diego, CA, USA, 22- 27 March 2009.

[8] Tynan, R.; Marsh, D.; O'Kane, D.; O'Hare, G.M.P., "Agents for wireless sensor network power management", International Conference Workshops on Parallel Processing (ICPP 2005), vol., pp. 413-418, June 2005.

[9] Fok, C.-L.; Roman, G.-C.; Lu, C., "Mobile agent middleware for sensor networks: an application case study", Fourth International Symposium on Information Processing in Sensor Network.( IPSN 2005), pp. 382-387, April 2005.
[10] Hussain, S.; Shakshuki, E.; Matin, A.W., "Agent-based system architecture for wireless sensor networks", 20th International Conference on Advanced Information Networking and Applications (AINA 2006), vol.2, April 2006.

[11] Zhou Ying; Xiao Debao, "Mobile agent-based policy management for wireless sensor networks," Proceedings of 2005 International Conference on Wireless Communications, Networking and Mobile Computing, vol.2, pp. 1207-1210, 23-26 Sept. 2005.

[12] Akyildiz, I.; Su, W.; ankarasubramaniam, Y.; Cayirci, E. “A survey on sensor networks", IEEE Commun. Mag., 40, 102-114, 2002

[13] Yick, J.; Mukherjee, B.; Ghosal, D. "Wireless sensor network survey”, Comput. Netw., 52, 2292-2330, 2008.

[14] Chong, C.-Y.; Kumar, S.P. "Sensor networks: Evolution, opportunities, and challenges", Proc. IEEE, 91, 12471256, 2003.

[15] Sohraby, K.; Minoli, D.; Znati, T. "Wireless Sensor Networks: Technology, Protocols, and Applications", 1st ed.; John Wiley \& Sons, Inc: Hoboken, NJ, USA, pp. 3869, 2007.

[16] Zhao, F.; Guibas, L. "Wireless Sensor Networks", 1st ed.; Elsevier: San Francisco, CA, USA, pp. 9-16, 2004.

[17] Arampatzis, T.; Lygeros, J.; Manesis, S. "A survey of applications of wireless sensors and wireless sensor networks", In Proceedings of the 13th IEEE Mediterranean Conference on Control and Automation, Nicosia, Cyprus, pp. 719-724, June 27-29, 2005.

[18] García-Hernández, C.F.; Ibargüengoytia-González, P.H.; García-Hernández, J.; Pérez-Díaz, J.A. "Wireless sensor networks and applications: A survey", Int. J. Comput. Sci. Netw. Secur., 7, 264-273, 2007.

[19] W.R. Heinzelman, A. Chandrakasan, H. Balakrishnan, "Energy efficient communication protocol for wireless micro sensor networks", in: Hawaii International Conference on System Sciences (HICSS), 2000. 